\title{
Characterizations of Solutions for Vector Equilibrium Problems ${ }^{1,2}$
}

\author{
Q. H. AnsAri, ${ }^{3}$ I. V. Konnov, ${ }^{4}$ AND J. C. YAO $^{5}$ \\ Communicated by S. Schaible
}

\begin{abstract}
In this paper, we characterize the solutions of vector equilibrium problems as well as dual vector equilibrium problems. We establish also vector optimization problem formulations of set-valued maps for vector equilibrium problems and dual vector equilibrium problems, which include vector variational inequality problems and vector complementarity problems. The set-valued maps involved in our formulations depend on the data of the vector equilibrium problems, but not on their solution sets. We prove also that the solution sets of our vector optimization problems of set-valued maps contain or coincide with the solution sets of the vector equilibrium problems.
\end{abstract}

Key Words. Vector optimization problems, vector equilibrium problems, dual vector equilibrium problems, set-valued maps, characterization of solutions.

\section{Introduction}

Equilibrium problems, which include variational inequality, optimization, and complementarity problems as special cases, have been studied by Blum and Oettli (Refs. 1-2) and by Bianchi and Schaible (Ref. 3). Auchmuty (Ref. 4) introduced a variational principle for variational inequalities in finite-dimensional spaces. Blum and Oettli (Ref. 1) established variational

\footnotetext{
${ }^{1}$ In this research, the first and third author were supported by the NSC of the Republic of China. The second author was supported in part by RFBR Grant 01-01-00070.

${ }^{2}$ The authors are grateful to the referees for valuable suggestions and comments improving the original draft.

${ }^{3}$ Reader, Department of Mathematics, Aligarh Muslim University, Aligarh, India.

${ }^{4}$ Professor, Department of Applied Mathematics, Kazan State University, Kazan, Russia.

${ }^{5}$ Professor, Department of Applied Mathematics, National Sun Yat-Sen University, Kaohsiung, Taiwan, ROC.
} 
principles for equilibrium problems, which generalize the gap function (Refs. 5-6) used in connection with variational inequalities. Very recently, Chen et al. (Ref. 7) introduced the gap function for vector variational inequalities introduced by Giannessi (Ref. 8). Inspired by the notion of vector variational inequality, the equilibrium problem has been extended to vector-valued functions and is known as the vector equilibrium problem (VEP), which contains vector optimization problems, vector variational inequality problems, and vector complementarity problems as special case. This problem was studied by Ansari et al. (Refs. 9-10), Bianchi et al. (Ref. 11), Fu (Ref. 12), Hadjisavvas and Schaible (Ref. 13), Konnov (Ref. 14), Lee et al. (Ref. 15), and Oettli and Schläger (Refs. 16-17). But to date, it appears that no work has been done on the characterization of solutions for vector equilibrium problems. This paper is the first effort in this direction.

In Section 2, we give some preliminaries and formulations of our problems. In Section 3, we establish some characterizations of solutions for vector equilibrium problems by means of solving the zero inclusion problems for set-valued maps. We introduce also vector optimization problem (VOP) formulations of set-valued maps for the (VEP). We prove that the solution sets of our vector optimization problems contain or coincide with the solution set of the (VEP). Section 4 deals with the characterization of solutions for the dual vector equilibrium problem. We propose also suitable vector optimization problem formulations of set-valued maps for the dual vector equilibrium problem. Furthermore, we consider the relationships between the solution sets of these vector optimization problems and the (VEP).

\section{Preliminaries and Formulations}

Let $X$ and $Y$ be topological vector spaces, and let $C$ be a pointed closed convex proper cone in $Y$, with int $C \neq \varnothing$, where int $C$ denotes the interior of the set $C$. Then, $C$ induces a vector ordering in $Y$ setting, for all $x, y \in C$,

$x \leq y, \quad$ if and only if $y-x \in C$,

$x \not y, \quad$ if and only if $y-x \notin C$.

Since int $C \neq \varnothing$, we have also a weak order in $Y$ setting, for all $x, y \in C$,

$x<y, \quad$ if and only if $y-x \in \operatorname{int} C$,

$x \nless y, \quad$ if and only if $y-x \notin \operatorname{int} C$.

The orderings $\geq, \ngtr,>, \ngtr$ are defined similarly. 
Let $K$ be nonempty convex set in $X$, and let $f: K \times K \rightarrow Y$ be a bifunction satisfying

$$
f(x, x)=0, \quad \text { for all } x \in K .
$$

In this paper, we shall consider the vector equilibrium problem (VEP): find $x_{0} \in K$ such that

$$
f\left(x_{0}, y\right) \nless 0, \quad \text { for all } y \in K .
$$

Existence of solutions of this problem has been investigated in Refs. 11-13 and 16.

For the case of a moving cone, it has also been studied in Refs. 9 and 15. For a direct application of the (VEP), we refer to Ref. 14. We denote by $P$ the solution set of the (VEP).

It is well-known that the (VEP) is closely related to the following problem: find $x_{0} \in K$ such that

$$
f\left(y, x_{0}\right) \ngtr 0, \quad \text { for all } y \in K .
$$

This problem can be termed as the dual vector equilibrium problem (DVEP), Ref. 12. We denote by $D$ the solution set of the (DVEP).

Now, we mention some definitions and results which will be used in the sequel.

Definition 2.1. See Ref. 11. A bifunction $f: K \times K \rightarrow Y$ is called:

(a) monotone if, for all $x, y \in K$, $f(x, y)+f(y, x) \leq 0 ;$

(b) pseudomonotone if, for all $x, y \in K$, $f(x, y)>0$ implies $f(y, x)<0$.

Definition 2.2. See Ref. 11. A function $h: K \rightarrow Y$ is called:

(i) quasiconvex if, for all $\alpha \in Y$, the set

$L(\alpha)=\{x \in K: h(x) \leq \alpha\}$

is convex;

(ii) explicitly quasiconvex if $h$ is quasiconvex and, for all $x, y \in K$ such that $h(x)<h(y)$, we have

$h\left(z_{t}\right)<h(y), \quad$ for all $z_{t}=t x+(1-t) y$ and $t \in(0,1) ;$

(iii) hemicontinuous if, for any $x, y \in K$ and $t \in[0,1]$, the mapping $t \mapsto h(t x+(1-t) y)$ is continuous at $0^{+}$. 
We note that, if $h$ is quasiconvex, then the set $\{x \in K: h(x)<\alpha\}$ is also convex.

From Proposition 3.1 in Ref. 11, we obtain the following relationship between the solution sets of problems (VEP) and (DVEP).

Proposition 2.1. Let $K$ be a nonempty convex subset of a Hausdorff topological vector space $X$, and let $f: K \times K \rightarrow Y$ be a bifunction such that $f(x, x)=0$ for all $x \in K$. Then,

(i) Problem (1) implies problem (2) if $f$ is pseudomonotone.

(ii) Problem (2) implies problem (1) if $f(x, \cdot)$ is explicitly quasiconvex and $f(\cdot, y)$ is hemicontinuous for all $x, y \in K$.

We denote

$$
f(K, y)=\bigcup_{x \in K} f(x, y), \quad f(x, K)=\bigcup_{y \in K} f(x, y) .
$$

If $A$ is a nonempty subset of $Y$, then we set

$$
\begin{aligned}
& \max _{C} A:=\left\{a \in A \mid \text { there exists no } a^{\prime} \in A \text { such that } a^{\prime} \neq a \text { and } a^{\prime} \geq a\right\}, \\
& \min _{C} A:=\left\{a \in A \mid \text { there exists no } a^{\prime} \in A \text { such that } a^{\prime} \neq a \text { and } a^{\prime} \leq a\right\}, \\
& w-\max _{C} A:=\left\{a \in A \mid \text { there exists no } a^{\prime} \in A \text { such that } a^{\prime}>a\right\}, \\
& w-\min _{C} A:=\left\{a \in A \mid \text { there exists no } a^{\prime} \in A \text { such that } a^{\prime}<a\right\} .
\end{aligned}
$$

Note, for example, that it is possible that $\max _{C} A=\varnothing$.

\section{Characterizations of Solutions for (VEP)}

In this section, we aim to derive some characterizations of solutions for the vector equilibrium problem (VEP) defined by (1). We introduce also the vector optimization problem formulation of set-valued mappings for the vector equilibrium problem in the sense that there exists a set-valued map $F: K \rightarrow 2^{Y}$, depending on the data of the (VEP) but not on its solution set, such that the solution set of the (VEP) coincides with the solution set of the vector optimization problem (VOP), which is defined with the help of the map $F$. For instance, we can define the following (VOP):

$$
w-\min _{x \in K} F(x) ;
$$


that is, to find all $x_{0} \in K$ for which there exists $y_{0} \in F\left(x_{0}\right)$ such that

$$
y_{0} \in w-\min _{C} F(K)
$$

i.e.,

$$
F\left(x_{0}\right) \cap w-\min _{C} F(K) \neq \varnothing,
$$

where

$$
F(K)=\bigcup_{x \in K} F(x) .
$$

For the existence of solutions and other theoretical work on the (VOP) (3), we refer to Refs. 18-22 and references therein.

In order to formulate our first characterization, we define a set-valued map $\phi: K \rightarrow 2^{Y}$ as follows:

$$
\phi(x)=w-\min _{C} f(x, K), \quad \text { for all } x \in K .
$$

Let

$$
\operatorname{dom}(\phi)=\{x \in K: \phi(x) \neq \varnothing\} .
$$

Lemma 3.1. For each $x \in K$, we have that

$$
z \in \phi(x) \text { implies } z \ngtr 0 .
$$

Proof. For contradiction, assume that there exists $z \in \phi(x)$ such that $z>0$. Then, there exists $y \in K$ such that

$$
f(x, y)=z>0=f(x, x),
$$

which is a contradiction, since

$$
f(x, y) \in w-\min _{C} f(x, K) .
$$

We associate to the (VEP) the following (VOP) for set-valued map:

$$
w-\max _{x \in K} \phi(x) ;
$$

that is, to find all $x_{0} \in K$ for which there exists $y_{0} \in \phi\left(x_{0}\right)$ such that $y_{0} \in$ $w-\max _{C} \phi(K)$, i.e.,

$$
\phi\left(x_{0}\right) \cap w-\max _{C} \phi(K) \neq \varnothing,
$$


where

$$
\phi(K)=\bigcup_{x \in K} \phi(x)
$$

We denote by $Q_{\phi}$ the solution set of problem (5).

\section{Theorem 3.1.}

(i) $x_{0} \in K$ is a solution of the (VEP) if and only if $0 \in \phi\left(x_{0}\right)$.

(ii) $P \subseteq Q_{\phi}$.

Proof. Suppose that $x_{0}$ solves the (VEP). Then,

$$
f\left(x_{0}, y\right) \not \neq, \quad \text { for all } y \in K \text {. }
$$

Assume that $0 \notin \phi\left(x_{0}\right)$; then, there exists $z \in K$ such that

$$
f\left(x_{0}, z\right)<0 \text {, }
$$

which is a contradiction.

Conversely, let $0 \in \phi\left(x_{0}\right)$. Assume that $x_{0}$ does not solve the (VEP); then, there exists $y \in K$ such that $f\left(x_{0}, y\right)<0$. This implies that $0 \notin \phi\left(x_{0}\right)$, a contradiction. This proves the first part of the theorem.

Next, let $x_{0} \in P$. Then, $0 \in \phi\left(x_{0}\right)$ due to part (i). It now follows from (4) that $x_{0} \in Q_{\phi}$, as desired.

We remark that the inclusion in Theorem 3.1(ii) can be strict as the following example shows.

\section{Example 3.1. Let}

$$
X=Y=R \quad \text { and } \quad C=[0, \infty) .
$$

Let

$$
f(x, y)=\sin (x-y), \quad \text { for all } x, y \in R .
$$

Then, for each $x \in R, f(x, R)=[-1,1]$. Hence, for each $x \in R, \phi(x)=\{-1\}$. It is clear that $P=\varnothing$ and $Q_{\phi}=R$.

Let $Y$ be a locally convex space, and let $Y^{*}$ denote the topological dual space of $Y$; then, we can define the dual cone

$$
C^{*}:=\left\{\lambda \in Y^{*} \mid\langle\lambda, y\rangle \geq 0, \text { for all } y \in Y\right\}
$$

to $C$. Since int $C \neq \varnothing$ and $C \neq Y$, we have $C^{*} \neq\{0\}$; besides, $C^{*}$ has a weakly* compact base; i.e., there exists $B \subseteq C^{*}, B$ is convex, weakly* compact, such 
that $0 \notin B$ and $C^{*}=\bigcup_{t \geq 0} t B$. We fix such a base and set

$$
\sigma(u):=\max _{t \in B}(t, u), \quad \text { for all } u \in Y,
$$

(see Ref. 16). Then, for all $u \in Y$,

$$
\begin{array}{ll}
u<0 \Leftrightarrow \sigma(u)<0, & u \leq 0 \Leftrightarrow \sigma(u) \leq 0, \\
u \nless 0 \Leftrightarrow \sigma(u) \geq 0, & u \neq 0 \Leftrightarrow \sigma(u)>0 .
\end{array}
$$

In order to formulate our second characterization, we define the setvalued maps $Z: K \rightarrow 2^{K}$ and $\varphi: K \rightarrow 2^{Y}$ as follows:

$$
\begin{aligned}
& Z(x):=\{y \in K \mid \sigma(f(x, y)) \leq \sigma(f(x, z)), \text { for all } z \in K\}, \\
& \varphi(x):=f(x, Z(x)) .
\end{aligned}
$$

Lemma 3.2. For each $x \in K$, we have that

$$
z \in \varphi(x) \text { implies } z \leq 0 \text {. }
$$

Proof. By definition, if $z \in \varphi(x)$, then there exists $y \in K$ such that $z=$ $f(x, y)$. Since $y \in Z(x)$, we have

$$
\sigma(f(x, y)) \leq \sigma(f(x, x))=0 .
$$

From (6), it now follows that

$$
z=f(x, y) \leq 0 .
$$

We now define the following (VOP) for set-valued map:

$$
\max _{x \in K} \varphi(x)
$$

that is, to find all $x_{0} \in K$ for which there exists $y_{0} \in \varphi\left(x_{0}\right)$ such that $y_{0} \in$ $\max _{C} \varphi(K)$, i.e.,

$$
\varphi\left(x_{0}\right) \cap \max _{C} \varphi(K) \neq \varnothing .
$$

We denote by $Q_{\varphi}$ the solution set of problem (8).

\section{Theorem 3.2.}

(i) $x_{0} \in K$ is a solution of the (VEP) if and only if $0 \in \varphi\left(x_{0}\right)$.

(ii) If the solution set of the (VEP) is nonempty, then $P=Q_{\varphi}$.

Proof. Suppose that $x_{0}$ solves the (VEP). Then,

$$
f\left(x_{0}, y\right) \not 0, \quad \text { for all } y \in K,
$$


or due to (6),

$$
\sigma\left(f\left(x_{0}, y\right)\right) \geq 0, \quad \text { for all } y \in K .
$$

Therefore,

$$
\sigma\left(f\left(x_{0}, x_{0}\right)\right)=0 \leq \sigma\left(f\left(x_{0}, y\right)\right), \quad \text { for all } y \in K,
$$

and we conclude that $x_{0} \in Z\left(x_{0}\right)$. It follows that

$$
0=f\left(x_{0}, x_{0}\right) \in \varphi\left(x_{0}\right) .
$$

Conversely, let $0 \in \varphi\left(x_{0}\right)$. Assume that $x_{0}$ does not solve the VEP; then, there exists $y \in K$ such that $f\left(x_{0}, y\right)<0$. Now, (6) gives

$$
\sigma\left(f\left(x_{0}, y\right)\right)<0
$$

and

$$
\sigma\left(f\left(x_{0}, y^{\prime}\right)\right) \leq \sigma\left(f\left(x_{0}, y\right)\right)<0, \quad \text { for all } y^{\prime} \in Z\left(x_{0}\right) .
$$

Again, from (6), we have

$$
f\left(x_{0}, y^{\prime}\right)<0, \quad \text { for all } y^{\prime} \in Z\left(x_{0}\right) ;
$$

i.e. $0 \notin \varphi\left(x_{0}\right)$, a contradiction. This proves part (i) of the theorem.

Next, let $P \neq \varnothing$. Take any $x_{0} \in P$; then,

$$
0=f\left(x_{0}, x_{0}\right) \in \varphi\left(x_{0}\right),
$$

due to (i). From (7), we have that, for each $x \in K$ and for all $z \in \varphi(x)$,

$$
z \leq f\left(x_{0}, x_{0}\right)=0 .
$$

If there exists $z^{\prime} \in \varphi(x), z^{\prime} \neq 0$ such that $z^{\prime} \geq 0$, then we must have

$$
z^{\prime} \geq 0 \quad \text { and } \quad z^{\prime} \leq 0
$$

that is,

$$
0 \neq z^{\prime} \in C \cap(-C) .
$$

This contradicts that $C$ is a pointed one. Therefore, $x_{0} \in Q_{\varphi}$.

Conversely, take any $x_{0} \in Q_{\varphi}$. By the definition, there exists $y_{0} \in \varphi\left(x_{0}\right)$ such that $y_{0} \in \max _{C} \varphi(K)$. From (7), it follows that $y_{0} \leq 0$. For contradiction, assume that $0 \notin \varphi\left(x_{0}\right)$; then, $y_{0} \neq 0$. Since $P \neq \varnothing$, there exists $x^{*} \in P$, but $0 \in$ $\varphi\left(x^{*}\right)$ due to (i). Therefore, there exists $0 \in \varphi(K), 0 \neq y_{0}$, and $y_{0} \leq 0$. This contradicts that $y_{0} \in \max _{C} \varphi(K)$. Thus, assertion (ii) is also true and the proof is completed.

We remark that, in Theorem 3.2(ii), it is possible that $P$ is empty and $Q_{\varphi}$ is not empty by again considering Example 3.1. 


\section{Characterizations of Solutions for (DVEP)}

In this section, we shall derive some characterizations of solutions for the dual vector equilibrium problem (DVEP) defined by (2). Again, we will introduce some suitable vector optimization problem formulations of setvalued maps for the dual vector equilibrium problem.

We define first a set-valued map $\phi^{*}: K \rightarrow 2^{Y}$ by means of

$$
\phi^{*}(x)=w-\max _{C} f(K, x), \quad \text { for all } x \in K,
$$

and we associate to the (VEP) the following (VOP):

$$
w-\min _{x \in K} \phi^{*}(x) ;
$$

that is, to find all $x_{0} \in K$ for which there exists $y_{0} \in \phi^{*}\left(x_{0}\right)$ such that

$$
y_{0} \in w-\min _{C} \phi^{*}(K),
$$

i.e.,

$$
\phi^{*}\left(x_{0}\right) \cap w-\min _{C} \phi^{*}(K) \neq \varnothing .
$$

The set-value map (9) generalizes the gap function, introduced and studied in Ref. 7 for weak vector variational inequalities. We denote by $Q_{\phi^{*}}$ the solution set of problem (10). Note that, in the sense of vector optimization for set-valued maps, the (VOP) (5) and (10) are dual to each other; see Ref. 20 and references therein. Therefore, the (VOP) (10) is closely related to the dual formulation (2) of the (VEP). Similar to the proof of Theorem 3.1, we can get the following characterization of solution for the dual vector equilibrium problem.

\section{Proposition 4.1.}

(i) $x_{0} \in K$ is a solution of the (DVEP) if and only if $0 \in \phi^{*} Q\left(x_{0}\right)$.

(ii) $D \subseteq Q_{\phi^{*}}$.

Combining Propositions 2.1 and 4.1, we can obtain some existence results for the vector equilibrium problem. Let us first recall the following defintion.

Definition 4.1. A bifunction $f: K \times K \rightarrow Y$ is called bipseudomonotone if $f$ and $-f$ are pseudomonotone.

Example 4.1. Let

$$
X=Y=R^{2}, \quad K=[0,1] \times[0,1], \quad C=R_{+}^{2} .
$$


We define two bifunctions $f_{1}, f_{2}: K \times K \rightarrow Y$ by

$$
\begin{aligned}
& f_{1}(x, y)=\left(x_{1}-y_{1}, x_{2}^{2}-y_{2}^{2}\right), \\
& f_{2}(x, y)=\left(x_{2}\left(x_{1}-y_{1}\right), y_{2}\left(x_{2}-y_{2}\right)\right),
\end{aligned}
$$

where

$$
x=\left(x_{1}, x_{2}\right) \text { and } y=\left(y_{1}, y_{2}\right) .
$$

Then $f_{1}$ and $f_{2}$ are bipseudomonotone. Since

$$
f_{1}(x, y)+f_{1}(y, x)=0,
$$

for all $x, y \in K, f_{1}$ is also monotone. But $f_{2}$ is not monotone, because for $x=$ $(1,1)$ and $y=(1 / 2,1 / 2)$, we have

$$
f_{2}(x, y)+f_{2}(y, x)=(1 / 4,-1 / 4) \not 0 .
$$

Now, we can state the following existence results for the vector equilibrium problem.

Corollary 4.1. Let $K$ be a nonempty convex subset of a Hausdorff topological vector space $S$, and let $f: K \times K \rightarrow Y$ be a bifunction such that $f(x, x)=0$, for all $x \in K$.

(i) If $f$ is pseudomonotone and $x_{0} \in P$, then $0 \in \phi^{*}\left(x_{0}\right)$.

(ii) If $0 \in \phi^{*}\left(x_{0}\right), x_{0} \in K$, and either $-f$ is pseudomonotone, or $f(x, \cdot)$ is explicitly quasiconvex and $f(\cdot, y)$ is hemicontinuous for all $x, y \in$ $K$, then $x_{0} \in P$.

(iii) If $f$ is pseudomonotone, then $P \subseteq Q_{\phi^{*}}$.

Corollary 4.2. Let $K$ be a nonempty convex subset of a Hausdorff topological vector space $X$, and let $f: K \times K \rightarrow Y$ be a bifunction such that $f(x, x)=0$, for all $x \in K$. Suppose that at least one of the following assumptions hold:

(i) $f$ is a bipseudomonotone;

(ii) $f$ is pseudomonotone, $f(x, \cdot)$ is explicitly quasiconvex, and $f(\cdot, y)$ is hemicontinuous for all $x, y \in K$.

Then, $x_{0} \in K$ solves the (VEP) (1) if and only if $0 \in \phi^{*}\left(\mathrm{x}_{0}\right)$.

By analogy with the second characterization from Section 3, we can define the following characterization of solution for the dual vector equilibrium problem. We also present another vector optimization problem (VOP), which guarantees for the solution sets of the (VEP) and (VOP) to coincide. From now on, we suppose that $Y$ is a locally convex Hausdorff topological 
vector space. We define the set-valued maps $Z^{*}: K \rightarrow 2^{K}$ and $\varphi^{*}: K \rightarrow 2^{Y}$ as follows:

$$
\begin{aligned}
& Z^{*}(x):=\left\{y \in K \mid \sigma^{*}(f(y, x)) \geq \sigma^{*}(f(z, x)), \text { for all } z \in K\right\}, \\
& \varphi^{*}(x):=f\left(Z^{*}(x), x\right),
\end{aligned}
$$

where

$$
\sigma^{*}(y):=\min _{t \in B}(t, y), \quad \text { for all } y \in Y,
$$

$B$ is a base for $C^{*}$. Then (see e.g., Ref. 16), for all $u \in Y$, we have the following results (cf. (6)):

$$
\begin{array}{ll}
u>0 \Leftrightarrow \sigma^{*}(u)>0, & u \geq 0 \Leftrightarrow \sigma^{*}(u) \geq 0, \\
u \ngtr 0 \Leftrightarrow \sigma^{*}(u) \leq 0, & u \neq 0 \Leftrightarrow \sigma^{*}(u)<0 .
\end{array}
$$

We now define the following (VOP) for set-valued map:

$$
\min _{x \in K} \varphi^{*}(x) ;
$$

that is, to find all $x_{0} \in K$ for which there exists $y_{0} \in \varphi^{*}\left(x_{0}\right)$ such that $y_{0} \in$ $\min _{C} \varphi^{*}(K)$, i.e.,

$$
\varphi^{*}\left(x_{0}\right) \cap \min _{C} \varphi^{*}(K) \neq \varnothing .
$$

We denote by $Q_{\varphi^{*}}$ the solution set of problem (11).

\section{Proposition 4.2.}

(i) $x_{0} \in K$ is a solution of the (DVEP) if and only if $0 \in \varphi^{*}\left(x_{0}\right)$.

(ii) If the solution set of the (DVEP) is nonempty, then $D=Q_{\varphi^{*}}$.

Combining Propositions 2.1 and 4.2, we obtain the following chracterization of solution for the (VEP).

Corollary 4.3. Let all the assumptions of Corollary 4.2 hold. Then:

(i) $x_{0} \in K$ is a solution of the (VEP) if and only if $0 \in \varphi^{*}\left(x_{0}\right)$.

(ii) If the solution set of the (VEP) is nonempty, then $P=Q_{\varphi^{*}}$.

\section{References}

1. Blum, E., and Oetrli, W., Variational Principles for Equilibrium Problems, Parametric Optimization and Related Topics III, Edited by J. Guddad et al., Peter Lang, Frankfurt am Main, Germany, pp. 79-88, 1993. 
2. Blum, E., and Oettli, W., From Optimization and Variational Inequalities to Equilibrium Problems, The Mathematics Student, Vol. 63, pp. 123-145, 1994.

3. Bianchi, M., and Schaible, S., Generalized Monotone Bifunctions and Equilibrium Problems, Journal of Optimization Theory and Applications, Vol. 90, pp. 31-43, 1996.

4. Auchmuty, G., Variational Principles for Variational Inequalities, Numerical Functional Analysis and Optimization, Vol. 10, pp. 863-874, 1989.

5. Auslender, A., Optimisation: Méthodes Numériques, Masson, Paris, France, 1976.

6. Hearn, D. W., The Gap Function of a Convex Program, Operations Research Letters, Vol. 1, pp. 67-71, 1982.

7. Chen, G. Y., Goh, C. J., and YAng, X. Q., On Gap Functions for Vector Variational Inequalities, Vector Variational Inequalities Vector Equilibria: Mathematical Theories, Edited by F. Giannessi, Kluwer Academic Publishers, Dordrecht, Netherlands, pp. 55-72, 2000.

8. Giannessi, F., Theorems of the Alternative, Quadratic Programs, and Complementarity Problems, Variational Inequalities and Complementarity Problems, Edited by R. W. Cottle, F. Giannessi, and J. L. Lions, John Wiley and Sons, New York, NY, pp. 151-186, 1980.

9. Ansari, Q. H., Vector Equilibrium Problems and Vector Variational Inequalities, Vector Variational Inequalities and Vector Equilibria: Mathematical Theories, Edited by F. Giannessi, Kluwer Academic Publishers, Dordrecht, Netherlands, pp. $1-15,2000$.

10. Ansari, Q. H., Oettli, W., and Schläger, D., A Generalization of Vectorial Equilibria, Mathematical Methods of Operations Research, Vol. 46, pp. 147152, 1997.

11. Bianchi, M., Hadjisavvas, N., and Schaible, S., Vector Equilibrium Problems with Generalized Monotone Bifunctions, Journal of Optimization Theory and Applications, Vol. 92, pp. 527-542, 1997.

12. Fu, J., Simultaneous Vector Variational Inequalities and Vector Implicit Complementarity Problems, Journal of Optimization Theory and Applications, Vol. 93, pp. 141-151, 1997.

13. Hadjisavvas, N., and Schaile, S., From Scalar to Vector Equilibrium Problems in the Quasimonotone Case, Journal of Optimization Theory and Applications, Vol. 96, pp. 297-305, 1998.

14. Konnov, I. V., Combined Relaxation Method for Solving Vector Equilibrium Problems, Russian Mathematics (Izvestiya Vuzov Math), Vol. 39, pp. 51-59, 1995.

15. LeE, G. M., Kim, D. S., and Lee, B. S., On Noncooperative Vector Equilibrium, Indian Journal of Pure and Applied Mathematics, Vol. 27, pp. 735-739, 1996.

16. Oettli, W., A Remark on Vector-Valued Equilibria and Generalized Monotonicity, Acta Mathematica Vietnamica, Vol. 22, pp. 213-221, 1997.

17. Oettli, W., and Schläger, D., Generalized Vectorial Equilibria and Generalized Monotonicity, Functional Analysis with Current Applications in Science, Technology, and Industry, Edited by M. Brokate and A. H. Siddiqi, 
Pitman Research Notes in Mathematics, Longman, Essex, England, Vol. 377, pp. 145-154, 1998.

18. Corley, H. W., Existence and Lagrange Duality for Maximization of Set-Valued Functions, Journal of Optimization Theory and Applications, Vol. 54, pp. 489$501,1987$.

19. Corley, H. W., Optimality Conditions for Maximization of Set-Valued Functions, Journal of Optimization Theory and Applications, Vol. 58, pp. 1-10, 1988.

20. Li, Z. F., and Chen, G. Y., Lagrangian Multipliers, Saddle Points, and Duality in Vector Optimization of Set-Valued Maps, Journal of Mathematical Analysis and Applications, Vol. 215, pp. 297-316, 1997.

21. Lin, L. J., Optimization of Set-Valued Functions, Journal of Mathematical Analysis and Applications, Vol. 186, pp. 30-51, 1994.

22. Luc, D. T., Theory of Vector Optimization, Lecture Notes in Economics and Mathematical System, Springer Verlag, New York, NY, Vol. 319, 1989. 